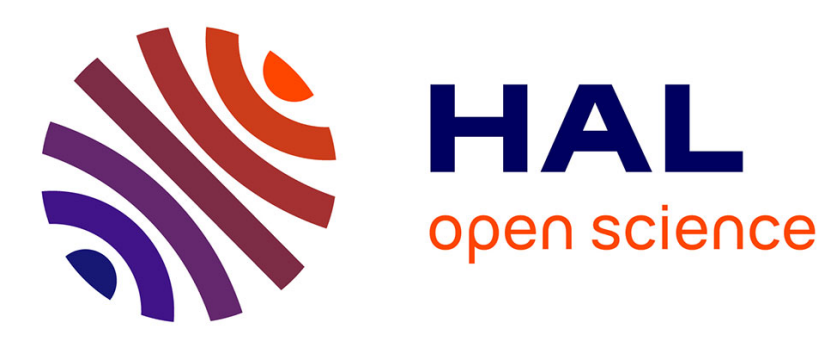

\title{
Tracking Level Set Representation Driven by a Stochastic Dynamics
}

\author{
Christophe Avenel, Etienne Mémin, Patrick Pérez
}

\section{To cite this version:}

Christophe Avenel, Etienne Mémin, Patrick Pérez. Tracking Level Set Representation Driven by a Stochastic Dynamics. International Conference on Curves and Surfaces, Jul 2010, Avignon, France. pp.130-141, 10.1007/978-3-642-27413-8_8. hal-00694591

\section{HAL Id: hal-00694591 \\ https://hal.inria.fr/hal-00694591}

Submitted on 4 May 2012

HAL is a multi-disciplinary open access archive for the deposit and dissemination of scientific research documents, whether they are published or not. The documents may come from teaching and research institutions in France or abroad, or from public or private research centers.
L'archive ouverte pluridisciplinaire HAL, est destinée au dépôt et à la diffusion de documents scientifiques de niveau recherche, publiés ou non, émanant des établissements d'enseignement et de recherche français ou étrangers, des laboratoires publics ou privés. 


\title{
Tracking Level Set Representation Driven by a Stochastic Dynamics
}

\author{
Christophe Avenel ${ }^{1}$, Etienne Mémin ${ }^{2}$, and Patrick Pérez ${ }^{3}$ \\ 1 Université Rennes 1 \\ cavenel@inria.fr \\ 2 Inria \\ etienne.memin@inria.fr \\ 3 Technicolor Corporate Research \\ patrick.perez@technicolor.com
}

\begin{abstract}
We introduce a non-linear stochastic filtering technique to track the state of a free curve from image data. The approach we propose is implemented through a particle filter, which includes color measurements characterizing the target and the background respectively. We design a continuous-time dynamics that allows us to infer inter-frame deformations. The curve is defined by an implicit level-set representation and the stochastic dynamics is expressed on the level-set function. It takes the form of a stochastic partial differential equation with a Brownian motion of low dimension. Specific noise models lead to the traditional level set evolution law based on mean curvature motions, while other forms lead to new evolution laws with different smoothing behaviors. In these evolution models, we propose to combine local photometric information, some velocity induced by the curve displacement and an uncertainty modeling of the dynamics. The associated filter capabilities are demonstrated on various sequences with highly deformable objects.
\end{abstract}

Keywords: Tracking, Particle filtering, Level set, Continuous dynamic.

\section{Introduction}

The video tracking of an interface between two regions is a central process in numerous domains like medical imaging, meteorology or traffic control. Despite the many solutions proposed, no optimal solution exists yet for state variables defined on large dimensional spaces such as curves.

In order to deal with regions undergoing a complex deformation along time and potentially involving topological changes, we will confine ourself to a level set representation of the region boundaries. This representation has the great advantage to formulate the curve evolution within an Eulerian framework which avoids the definition of splitting/merging stratagem of Lagrangian splines curve representations when the curve is subject to topological changes.

Many tracking approaches proposed so far for the tracking of a level set curve representation are often defined as techniques implementing successive 
almost independent detection processes on each image of the image sequence 3778 191112 . Even if those techniques includes a temporal initialization strategy, they cannot truly be considered as tracking processes, as they do not guaranty any temporal coherence of the curve point trajectory. Such temporal incoherences are all the more pregnant that ambiguities due to clutter noise or illumination variations are observed. In addition to this, the few probabilistic techniques that have been proposed so far in the literature for curve tracking 5114 are built upon adhoc linear models of the curve evolution law which limits them to the tracking of objects undergoing small or quasi-rigid deformations.

In the solution we propose, the evolution law is defined as a continuous-time stochastic dynamical model. The tracking is formulated as a stochastic filtering problem in which the available photometric data are filtered by such stochastic evolution laws. Stochastic filtering in high dimensional spaces is excruciatingly difficult to implement with particle or ensemble filters due to obvious sampling difficulties of high dimensional pdf., it is hence very important to devise dynamics that are the most accurate as possible. In the same time, we have to circumscribe the space of the random deformations applied on the curve in order to be able to efficiently draw samples, but also to make possible an efficient exploration of the considered curve's state space. To that end, the curve dynamics on which we rely is formulated as a stochastic transport equation defined directly on the implicit surface function. It includes constant displacement uncertainties along the curve tangent and normal directions. The transport velocity at each point of the curve is inferred from its past trajectory. This transport velocity field is computed through an additional vectorial level set function maintaining along time the correspondences between the current curve's points location and their original positions. The dynamics describing the evolution of this auxiliary function incorporates the uncertainties on the curve deformations as well. The curve dynamics is supplemented with a local photometric information that takes the form of a data-driven force, in order to guide more efficiently the curve prediction toward the next image observation.

\section{Continuous-Time Dynamical Model of Level Set and Filtering}

This section first recalls the general principles of particle filtering and presents the level set framework. Built on these ingredients, the proposed approach is then detailed.

\subsection{Particle Filter}

In this subsection, we introduce the filtering method we are using in the rest of the paper. The corresponding filters, called particle filters, are very general in the sense they enable coping with nonlinear dynamics and nonlinear measurement with additive eventually non-Gaussian noises. Denoting by $\mathbf{x}_{0: k}$ the trajectory from the initial time up to the current time instant $k$ of the hidden Markov 
process we want to estimate from the whole set of past observations $\mathbf{z}_{1: k}$, a recursive expression of the filtering distribution $p\left(\mathbf{x}_{0: k} \mid \mathbf{z}_{1: k}\right)$ can be obtained from Bayes' law and the assumption that the measurements depends only on the current state:

$$
p\left(\mathbf{x}_{0: k} \mid \mathbf{z}_{1: k}\right)=p\left(\mathbf{x}_{0: k-1} \mid \mathbf{z}_{1: k-1}\right) \frac{p\left(\mathbf{z}_{k} \mid \mathbf{x}_{k}\right) p\left(\mathbf{x}_{k} \mid \mathbf{x}_{k-1}\right)}{p\left(\mathbf{z}_{k} \mid \mathbf{z}_{1: k-1}\right)} .
$$

Particle filtering techniques implement an approximation of this density, using a sum of $N$ Diracs centered on hypothesized locations in the state space. At each one of these locations - called particles - is assigned a weight $w_{k}^{(i)}$ describing his relevance. This approximation can be formulated as

$$
p\left(\mathbf{x}_{0: k} \mid \mathbf{z}_{1: k}\right) \approx \sum_{i=1}^{N} w_{k}^{(i)} \delta_{\mathbf{x}_{0: k}}\left(\mathbf{x}_{0: k}\right) .
$$

It is impossible to simulate the samples directly from this unknown distribution. Particles are thus simulated from a proposal distribution $\pi\left(\mathbf{x}_{0: k} \mid \mathbf{z}_{1: k}\right)$. This distribution, called the importance distribution, approximates the true filtering distribution. Each sample is then weighted using the ratio between the two distributions. The value of $w_{k}^{(i)}$ accounts for the deviation with respect to the unknown true distribution.

As a result, the target distribution will be fairly sampled by the particles $\mathbf{x}_{0: k}^{(i)}$ weighted by weights $w_{k}^{(i)}$, defined as

$$
w_{k}^{(i)}=\frac{p\left(\mathbf{x}_{0: k}^{(i)} \mid \mathbf{z}_{1: k}\right)}{\pi\left(\mathbf{x}_{0: k}^{(i)} \mid \mathbf{z}_{1: k}\right)} .
$$

To get the best efficiency the approximation needs obviously to be the closest as possible to the true distribution. However, any importance function can be chosen, with the only restriction that its support contains the target density's one. The importance ratio can be recursively computed assuming the importance density can be written in the following recursive form:

$$
\pi\left(\mathbf{x}_{0: k} \mid \mathbf{z}_{1: k}\right)=\pi\left(\mathbf{x}_{0: k-1} \mid \mathbf{z}_{1: k-1}\right) \pi\left(\mathbf{x}_{k} \mid \mathbf{z}_{1: k}, \mathbf{x}_{0: k-1}\right)
$$

As $p\left(\mathbf{z}_{k} \mid \mathbf{z}_{1: k-1}\right)$ is the same for every particle, it can be removed from relation (1), which leads to a general recursive update formulation of the weights at the current time when the measurement $\mathbf{z}_{k}$ becomes available:

$$
w_{k}^{(i)} \propto w_{k-1}^{(i)} \frac{p\left(\mathbf{z}_{k} \mid \mathbf{x}_{k}^{(i)}\right) p\left(\mathbf{x}_{k}^{(i)} \mid \mathbf{x}_{k-1}^{(i)}\right)}{\pi\left(\mathbf{x}_{k}^{(i)} \mid \mathbf{x}_{0: k-1}^{(i)}, \mathbf{z}_{1: k}\right)} .
$$

Using (2) and the normalized weights, it is then easy to obtain marginals of the complete filtering density:

$$
p\left(\mathbf{x}_{k} \mid \mathbf{z}_{1: k}\right) \approx \sum_{i=1}^{N} w_{k}^{(i)} \delta_{\mathbf{x}_{k}}\left(\mathbf{x}_{k}\right) .
$$


Thus, by propagating the particles from time $k-1$ through the proposal density $\pi\left(\mathbf{x}_{k}^{(i)} \mid \mathbf{x}_{0: k-1}^{(i)}, \mathbf{z}_{1: k}\right)$, and by weighting the sampled states with $w_{k}^{(i)}$, we obtain a sampling of the filtering law.

Asymptotically, for a number of particles tending to infinity, convergence toward the Bayesian filtering distribution of various classes of particle filters has been demonstrated [4 with a rate of $1 / \sqrt{N}$. In practical implementations the number of particles is difficult to fix. The number of required particles to ensure the filter convergence depends on the state space dimension but also on the ability we have to draw samples in meaningful areas of this state space.

A resampling step of the particles is necessary to avoid the increase over time of the weight variance. Without this step, the number of significant particles decreases significantly along time. This procedure discards particles with weak weights, and duplicates particles with high weights.

When the proposal distribution is set to the dynamics, the weights updating rule (5) simplifies to the data likelihood $p\left(\mathbf{z}_{k} \mid \mathbf{x}_{k}^{(i)}\right)$. This particular instance of the particle filter is called the Bootstrap filter and constitutes the filtering method we will use in this study.

\subsection{Implicit Representation of the Curve}

In order to cope with curve's deformation of any kinds, it is essential to rely on a curve representation enabling easily the handling of topological changes arising when the region of interest splits apart in several separated components or on the contrary reassembles. The Level set formalism [11|15] has been specifically introduced in that goal to bypass the deficiency of splines based curves representation to manage such situations. In this representation the curve $\mathcal{C}_{t}$ at time $t$ is defined as the zero level set of a scalar function $\varphi(x, t): \Omega \times \mathbb{R}^{+} \rightarrow \mathbb{R}$ :

$$
\mathcal{C}_{t}=\{x \in \Omega \mid \varphi(x, t)=0\},
$$

where $\Omega$ stands for the image spatial domain. The implicit surface function, $\varphi$, is chosen so as to have for instance positive values inside the curve and negative values outside. A common choice for the implicit function is the signed distance function but any other surface whose level set fits the curve of interest is possible. The surface evolution is then defined in order to stick at all time to the contour dynamics. This representation has the great advantage to allow describing through a single implicit surface a set of non-intersecting closed curves. The main geometric features of the curve that will be needed for the curve evolution computation can be directly obtained from the implicit surface. In particular, the inward unit normal and the mean curvature are respectively given by:

$$
\boldsymbol{n}=\frac{\nabla \varphi}{\|\nabla \varphi\|} \text { and } \kappa=\operatorname{div}\left(\frac{\nabla \varphi}{\|\nabla \varphi\|}\right)=\frac{1}{\|\nabla \varphi\|}\left(\Delta \varphi-\nabla \varphi^{T} \nabla^{2} \varphi \nabla \varphi\right),
$$

where $\Delta \varphi$ and $\nabla^{2} \varphi$ denote the Laplacian and the Hessian of $\varphi$, respectively. 


\subsection{Stochastic Dynamics}

Efficient random sampling in high dimensional state space are known to be problematic. Some work have been done on Quasi-Random Sampling-High Dimensional Model Representation [6], but there is no optimal solution for random sampling in our case. In order to circumvent this problem, we will restrain the potential uncertainty on the curve's deformations to belong to a space of low dimension. To that end, the uncertainty on the curve deformation will be defined from two independent constant Brownian motions directed along the curve's normal and tangent:

$$
d \mathcal{C}_{t}=v_{n} \boldsymbol{n} d t+\sigma_{n} \boldsymbol{n} d B_{n, t}+\sigma_{\tau} \boldsymbol{n}^{\perp} d B_{\tau, t} .
$$

In this equation $d B_{n, t}$ and $d B_{\tau, t}$ denote the two Brownian motions, $\sigma_{n}$ and $\sigma_{\tau}$ are two diffusion coefficients, $\boldsymbol{n}$ is the unit vector normal to the curve and $v_{n}=\mathbf{v}^{T} \boldsymbol{n}$ is the projection on the curve's normal of a deterministic transport velocity field v. The random Brownian diffusion terms encode the curve deformation uncertainty. The deterministic transportation drift component will be further detailed in Section 2.4

The surface $\varphi$ can be used to express the deformation of the curve (9) on space $\Omega$ :

$$
d \mathcal{X}_{t}=w_{n}^{*} \frac{\nabla \varphi}{|\nabla \varphi|} d t+\sigma_{n} \frac{\nabla \varphi}{|\nabla \varphi|} d B_{n, t}+\sigma_{\tau} \frac{\nabla \varphi}{|\nabla \varphi|}^{\perp} d B_{\tau, t} .
$$

where $w_{n}^{*}$ is an extension on the whole image domain of the curve's drift strength component.

The curve at time $t$ is defined by construction through its implicit representation at time $t$ :

$$
\varphi(., t)=\varphi(., 0)+\int_{0}^{t} d \varphi(., s) .
$$

It is thus a function of the stochastic process $\mathcal{C}_{t}$.

As for a fixed point $x, \varphi(x, t)$ is a semi-martingale, the differential of $\varphi\left(\mathcal{X}_{\sqcup, \sqcup)}\right.$ has to be calculated through the Ito-Wentzell formula (differential of the composition of two stochastic process):

$$
d \varphi(x, t)=d \varphi_{t}(x)+\nabla \varphi^{T} d \mathcal{X}+\frac{1}{2} \sum_{i, j} d\left\langle\mathcal{X}_{t}^{i}, \mathcal{X}_{t}^{j}\right\rangle \frac{\partial^{2} \varphi}{\partial x_{i} \partial x_{j}}+\sum_{i} d\left\langle\frac{\partial \varphi}{\partial x_{i}}, \mathcal{X}_{t}^{i}\right\rangle
$$

It then leads to

$$
\begin{aligned}
d \varphi_{t}(x) & =b(y, t) d t+f(y, t) d B_{n, t} \\
& =-\nabla \varphi^{T} w_{n}^{*} d t-\frac{\sigma_{\tau}^{2} d t}{2}\left(\Delta \varphi-\frac{1}{|\nabla \varphi|^{2}} \nabla \varphi^{T} \Delta \varphi \nabla \varphi\right) \\
& +\frac{\sigma_{n}^{2} d t}{2}\left(\frac{1}{|\nabla \varphi|^{2}} \nabla \varphi^{T} \Delta \varphi \nabla \varphi\right)-\sigma_{n}|\nabla \varphi| d B_{n, t} .
\end{aligned}
$$


Compared to the classical deterministic level set differential, this expression introduces a Brownian component directed along the curve normal and an additional second-order smoothing term. The mean curvature component results from the introduction of the curve motion uncertainty along the curve tangent. It is worth noting that this formulation permits to interpret the mean curvature motion component as a consequence of the uncertainty one has on the curve's deformation along its tangent. This stochastic representation of the curve temporal evolution will enable us to draw samples of forecasted deformed curves.

\subsection{Velocity Computation by Keeping Curve's Point Correspondences}

The evolution law introduced in the previous section depends on a transport velocity field. This transport component could be derived from motion measurements estimated from the image sequence. However this solution has several drawbacks. It increases substantially the computational cost of the approach and requires the use of an external estimation technique. Furthermore, such a solution is not adapted to handle occlusions areas, where motion estimation is prone to errors. The use of such velocity measures would require thus the introduction of an additional occlusion detection mechanism and the definition of an alternative velocity field when occlusions occur. We propose instead to infer directly the velocity from each particle displacements, via a second implicit representation that keeps track of each point's starting location in the image plane. As proposed in 13 to keep this point correspondences, we introduce an additional vectorial level set $\boldsymbol{w}$ encoding on the level set domain the transportation of the curve's point location at the initial time. Keeping track of these backward point correspondences between the current evolving curve and a recent predecessor will allow us to derive an estimate of the curve's point velocity field.

More precisely, introducing the previous Cartesian coordinates of the curve's points location and encoding them through a vector-valued level set function $\psi^{k}: \mathbb{R}^{2} \times \mathbb{R}^{+} \rightarrow \mathbb{R}^{2}$ such that $\boldsymbol{\psi}^{k}(x, t)$ define the location that point $x \in \Omega$ at time $t \in[k, k-1]$ was occupying at previous instant $k-1$ :

$$
\psi^{k}(x, k-1)=x .
$$

This new level set function is intrinsically attached to the curve and undergoes deformations dictated by the stochastic curve's evolution law (9). Applying in the same way as previously the Ito formula, its differential reads:

$$
\begin{aligned}
d \psi^{i}(x, t) & =d \psi_{t}^{i}(x)+\left(\nabla \psi_{t}^{i}\right)^{T} d \mathcal{X}_{\sqcup} \\
& +\frac{1}{2} \sum_{i, j} d\left\langle\mathcal{X}_{t}^{i}, \mathcal{X}_{t}^{j}\right\rangle \frac{\partial^{2} \psi_{t}^{i}}{\partial x_{i} \partial x_{j}}+\sum_{i} d\left\langle\frac{\partial \psi_{t}^{i}}{\partial x_{i}}, \mathcal{X}_{t}^{i}\right\rangle=0 .
\end{aligned}
$$

In this case, the tangential component of the Brownian motion is not null. This function enables us to define the curve's transportation component (13) as

$$
\mathbf{v}(x, t) d t=\frac{1}{\Delta t}\left(x-E\left(\boldsymbol{\psi}^{k-1}(x, k-1) \mid \mathcal{C}_{k-1}\right)\right), t \in[k-1, k],
$$


where $E\left(\cdot \mid \mathcal{C}_{t}\right)$ denotes the expectation with respect to the the path of $\mathcal{C}_{t}$ up to time $t$ (formally the natural filtration associated to the process $\mathcal{C}_{t}$ ). This transportation velocity field is thus defined as a deterministic function computed from the realization of the curve (through its level set function $\varphi_{t}$ ) up to the previous instant $k-1$. Considering the particle approximation this velocity field is computed as

$$
\mathbf{v}(x, t) d t=\frac{1}{\Delta t}\left(x-\frac{1}{N} \sum_{i=1}^{N} w^{(i)} \boldsymbol{\psi}^{k-1,(i)}(x, k-1)\right), t \in[k-1, k],
$$

where $\boldsymbol{\psi}^{k-1,(i)}$ corresponds to the auxiliary level set distance function associated to particle $\varphi^{(i)}$ and $w^{(i)}$ its importance weight.

Let us note that as $\mathbf{v}(x, t)$ can only be used from instant $t=1$, we have to define a transport component for $t$ between 0 and 1 . This initial transportation component is set to the velocity field estimated from the two first images through an optical-flow estimator.

The velocity field is complemented with a local potential $F(\varphi)$ that corresponds to the Chan and Vese segmentation operator 2] extended to color histograms. It allows us to refine the tracking by taking into account color information, using local measurements that are inexpensive to compute1].

The two components are combined linearly with proportions $\beta(t) \in[0,1]$ and $1-\beta(t)$ respectively, yielding:

$$
v_{n}=\beta(t) \mathbf{v}^{T} \boldsymbol{n}+(1-\beta(t)) \partial_{\varphi} F(\varphi) .
$$

For our tracking purpose, the photometric component is especially helpful in the temporal vicinity of the second image, whereas the velocity component is more likely to be meaningful in the temporal vicinity of the first image. As a consequence we choose to change gradually the proportion of each components according to

$$
\beta(t)=t-k+1, t \in[k-1, k] .
$$

Equations (13 15) allows us to forecast instances of deformed curves and to sample the proposal distribution. The importance weights of these curve particles have to be updated from the data likelihood. We detail in the following section this likelihood and propose a technique for estimating the variances of the curve evolution law uncertainties.

\section{Measurement Models and Parameters Estimation}

\subsection{Likelihood Definition}

In bootstrap filters, the data likelihood associated to each particle directly determines its weight. It is therefore crucial for this distribution to be sufficiently

\footnotetext{
${ }^{1}$ As a consequence of the use of a data-driven force in the dynamics, the state space model is not anymore a classical hidden Markov model. It has been shown, however, that standard derivations that lead to filtering recursion can still be conducted with such models, leading to so-called conditional filters [1].
} 
discriminant in order to discard curves which are too distant from the intended result. To this end, we choose to define a likelihood that depends on the similarity between the color distributions inside the curve at times $t=0$ and $t=k$ respectively. This is a classical choice in literature [10. For each particle, it reads:

$$
p\left(\mathbf{z}_{t} \mid x_{t}^{(i)}\right) \propto \exp ^{-\lambda d\left(h_{0}, h_{k}^{(i)}\right)},
$$

where $d$ is the Bhattacharyya distance between $h_{0}$ the reference interior color histogram instantiated at time 0 and $h_{k}^{(i)}$ the interior color histogram associated to the $i$-th level set sample at time $k$, and $\lambda$ is a positive parameter. For discrete probability distributions $p$ and $q$ defined over the same domain $X$, the Bhattacharyya distance is defined as

$$
d(p, q)=\left(1-\sum_{x \in X} \sqrt{p(x) q(x)}\right)^{1 / 2}
$$

\subsection{Parameters Estimation}

The dynamics defined through equations (13]15] involves two diffusion coefficient related to the uncertainty associated to the curve motion. These parameters $\sigma_{n}$ and $\sigma_{\tau}$ can be derived from the displacements field between two consecutive images, as follows. Let $\mathbf{u}(x, t)$ be the displacement of point $x \in \Omega$ at time $k$ to its corresponding position at time $k+1$, according to the evolution of the implicit function $\varphi$ conditioned on the past observation. We assume that this displacement field is a noisy version of $\mathbf{v}$,

$$
\mathbf{u}(x, k)=\mathbf{v}(x, k) d t+\sigma_{n} \boldsymbol{n} d B_{n, t}+\sigma_{\tau} \boldsymbol{n}^{\perp} d B_{\tau, t},
$$

with noises along the normal and the tangent of $\varphi$ level-lines having same characteristics as those in (9). We are therefore making here the assumption that the noises associated with the level set displacement and the curve noises are collinear and have the same variances. Furthermore, we assume that the transport velocity field is such that $\mathbf{v}(x, t)=\mathbb{E}(\mathbf{u}(x, k)) / d t$. The empirical covariances with respect to the filtering law of this observed displacement along the curve normal and tangent provide an estimation of the noise variances $\sigma_{n}^{2}$ and $\sigma_{\tau}^{2}$ :

$$
\begin{gathered}
\sigma_{n}^{2}=\frac{1}{N-1} \sum_{i=1: N}\left(\frac{1}{n-1} \sum_{x \in \mathcal{C}^{(i)}} w^{(i)}((\mathbf{u}(x, k)-\mathbf{v}(x, k)) \cdot \boldsymbol{n}(x, t))^{2}\right) \\
\sigma_{\tau}^{2}=\frac{1}{N-1} \sum_{i=1: N}\left(\frac{1}{n-1} \sum_{x \in \mathcal{C}^{(i)}} w^{(i)}\left((\mathbf{u}(x, k)-\mathbf{v}(x, k)) \cdot \boldsymbol{n}^{\perp}(x, t)\right)^{2}\right) .
\end{gathered}
$$

For the time interval between the two first images, the values of these parameters are computed from the initial motion field used to initialize our filter. The next section shows results obtained for different kinds of image sequences. 


\section{Experiments and Results}

This section reports several tracking results obtained with our approach. We aim here at highlighting the main abilities of the method. Note that in all these experiments, the number of particles is fixed as $N=100$. Each curve initialization is performed manually, at the initial time. This initial curve will be systematically plotted for all the sequences tested.

\subsection{Interest of a Continuous-Time Stochastic Dynamics and Auxiliary Level-Set}

One of the main distinctive features of our approach is that it relies on a continuous-time stochastic dynamics. This allows the exploitation of temporal continuity even when deformations between successive images are drastic, as illustrated in the jellyfish sequence in Fig. 1 $(\mathrm{a}-\mathrm{e})$. This is in contrast with approaches relying on a succession of segmentation tasks. On the same sequence, for instance, the Chan-Vese segmentation method fails to recover an appropriate tracking of the delineated region (Fig. 1 : $\mathrm{f}-\mathrm{j}$ ). In this approach the segmentation process is initialized with the results obtained on the previous frame. There is no explicit handling of the possible deformation between two images. On the tracking results, in addition to the mean curve we plot through a white band a representation of the variance of the filtering. This aspect is further detailed in the next section.

Getting inter-frame tracking information is also of potential interest in context where successive images of the sequence are fairly distant in time, e.g., in meteorology and weather forecast.

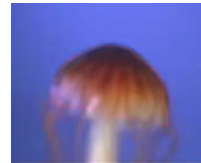

(a) $t=0$

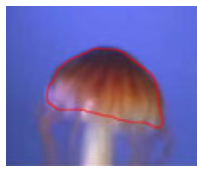

(f) $t=0$

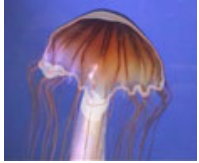

(b) $t=20$

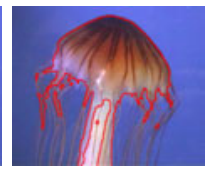

(g) $t=20$

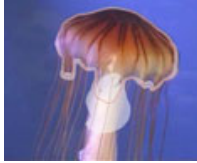

(c) $t=40$

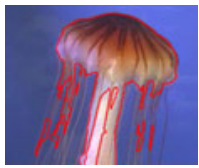

(h) $t=40$

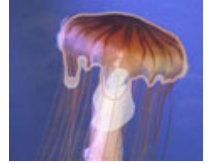

(d) $t=60$

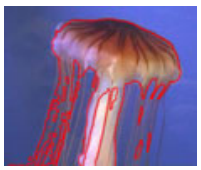

(i) $t=40$

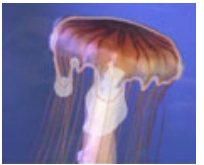

(e) $t=80$

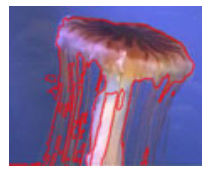

(j) $t=40$

Fig. 1. Tracking of a jellyfish, with a highly deformable body using our method (a - e) and deterministic Chan-Vese technique $(\mathrm{f}-\mathrm{j})$

\subsection{Variance Visualization and Analysis}

Beyond the tracking results provided by the weighted mean curve, local confidence assessment via local variance visualization (or analysis) is an interesting 
feature of our approach. The weighted set of implicit function samples provided by particle filtering permits such a visualization. The weighted local variance of the level set functions around the mean level set, which is obtained by computing $V=\sum_{i=1}^{N} w^{(i)}\left(\varphi^{(i)}-\bar{\varphi}\right)^{2}$, is here represented by a white band around the mean curve, the lower the variance, the narrower the uncertainty band. To illustrate this variance representation, we show results obtained on a second sequence (Fig. 2) showing a tiger running. In this sequence the colors of the background and the target are very close, which is an important source of ambiguities. We can observe in particular that for areas around the legs, the uncertainty is important. We observe that the results are good without using any external estimated motion field.

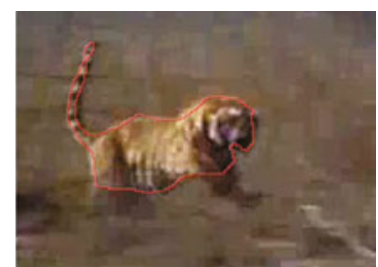

(a) $t=0$

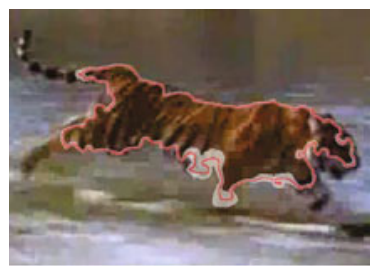

(d) $t=60$

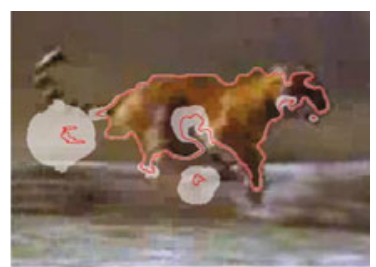

(b) $t=20$

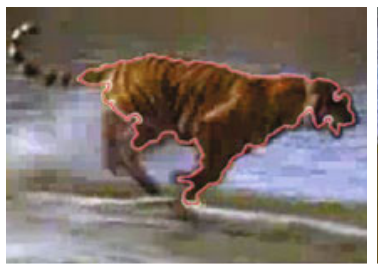

(e) $t=80$

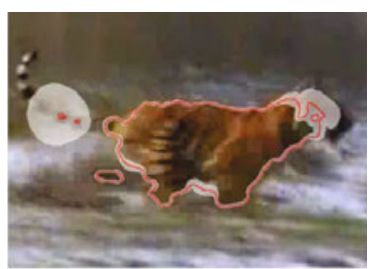

(c) $t=40$

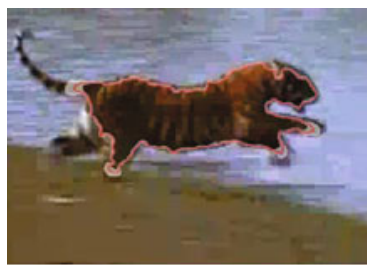

(f) $t=100$

Fig. 2. Tracking of running tiger with our particle filter on the space of implicit functions

To be able to access to an estimation of the tracking uncertainty is a great advantage of our technique. This should be of great interest in several application domains such as medical imaging in which the ability to quantify locally the quality of a result is essential for end-users.

\subsection{Occlusions Management}

One of the advantage provided by our transport velocity formulation compared to any optical flow measurements, is that it authorizes a natural handling of occlusion situations. As a matter of fact, no matter the region of interest be visible or not a velocity measure of the curve's is always available. This measure can thus be used at all instants without any distinction on the visibility or not of the considered point. There is here no need of empirical external occlusion detectors. For example, on Fig. 3. the person disappears on frames $d$ and $g$, but 


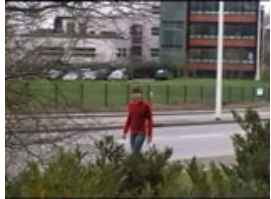

(a) $t=0$

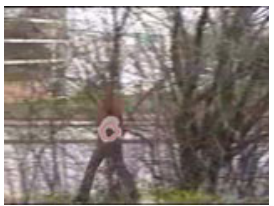

(e) $t=40$

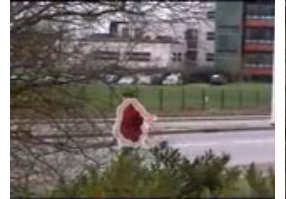

(b) $t=10$

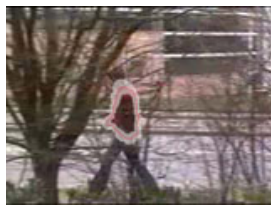

(f) $t=50$

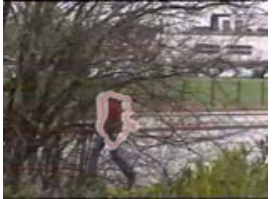

(c) $t=20$

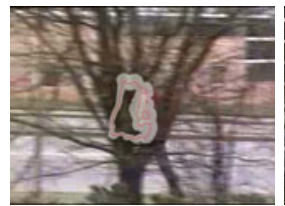

(g) $t=60$

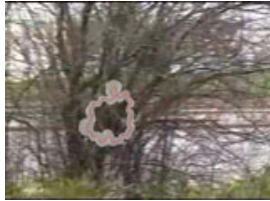

(d) $t=30$

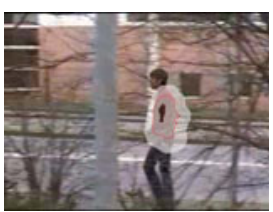

(h) $t=70$

Fig. 3. Example of occlusions on a body walking behind trees

tracking relocks on it after the occlusions end. We can observe that during the occlusions, the uncertainty is growing all around the curve, as the parameter $\sigma_{n}$ is growing.

As for the computational load. This approach can be straightforwardly on a multicore or grid computer as all the particles are independent. Only the weighting and the resampling requires communications between the processors. All these results have been run on a grid composed of 100 nodes, and the approach took nearly 5 minutes for a 100 images sequences. Let us note however that the level set has been implemented with a narrow band efficient implementation and could be hence much more faster than the present version.

\section{Conclusion}

In this paper we have proposed a probabilistic filtering method for the tracking of level sets. The underlying model combines discrete-time image measurements with a continuous-time stochastic dynamics. This dynamics relies on two different uncertainties on the curve motion, directed respectively along the curve normal and along the curve tangent. It also includes a transport vector field that combines an image-based force (related to local photometry) and a velocity induced by previous curve displacements and deformations. The measurement considered in this model are built from color histograms of the object delineated by the user at the initial time. The implementation of the filter is done via a particle filter whose proposal density amounts to simulating several steps of a discretized stochastic differential equation.

We have illustrated on several examples the interest of the continuous-time dynamics and of the estimation confidence assessment that proper stochastic filtering permits via the approximation of the filtering distribution. In particular, displaying the estimation uncertainty along the curve, which is done by computing the variance of each point of this curve, could be an interesting tool for, e.g., biology or meteorology imaging. Also, the ability to show inter-frame 
results is an other potential advantage that could be used to infer potential curve deformations between the two frames instant.

Finally, besides allowing to deal with occlusions, using the velocity of the curve could help predicting the evolution of this curve for a few frames ahead of time, which should be useful in various domains for forecast application.

\section{References}

1. Arnaud, E., Mémin, E.: Partial linear gaussian model for tracking in image sequences using sequential monte carlo methods. IJCV 74(1), 75-102 (2007)

2. Chan, T., Vese, L.: An active contour model without edges. In: Scale-Space Theories in Computer Vision, pp. 141-151 (1999)

3. Cremers, D., Soatto, S.: Motion competition: A variational framework for piecewise parametric motion segmentation. IJCV 62(3), 249-265 (2005)

4. Crisan, D., Doucet, A.: A survey of convergence results on particle filtering methods for practitioners. IEEE Transactions on Signal Processing 50(3), 736-746 (2002)

5. Dambreville, S., Rathi, Y., Tannenbaum, A.: Tracking deformable objects with unscented kalman filtering and geometric active contours. In: American Control Conference, pp. 1-6 (2006)

6. Feil, B., Kucherenko, S., Shah, N.: Comparison of monte carlo and quasi monte carlo sampling methods in high dimensional model representation. In: Proceedings of the 2009 First International Conference on Advances in System Simulation, pp. 12-17. IEEE Computer Society, Washington, DC, USA (2009)

7. Goldenberg, R., Kimmel, R., Rivlin, E., Rudzsky, M.: Fast geodesic active contours. IEEE Trans. on Image Processing 10(10), 1467-1475 (2001)

8. Kimmel, R., Bruckstein, A.M.: Tracking level sets by level sets: a method for solving the shape from shading problem. Comput. Vis. Image Underst. (1995)

9. Niethammer, M., Tannenbaum, A.: Dynamic geodesic snakes for visual tracking. In: CVPR (1), pp. 660-667 (2004)

10. Nummiaro, K., Koller-Meier, E., Gool, L.V.: An adaptive color-based particle filter. Image and Vision Computing 21(1), 99-110 (2003)

11. Osher, S., Sethian, J.A.: Fronts propagating with curvature-dependent speed: Algorithms based on Hamilton-Jacobi formulations. Journal of Computational Physics 79, 12-49 (1988)

12. Paragios, N., Deriche, R.: Geodesic active regions: a new framework to deal with frame partition problems in computer vision. J. of Visual Communication and Image Representation 13, 249-268 (2002)

13. Pons, J.P., Hermosillo, G., Keriven, R., Faugeras, O.: Maintaining the point correspondence in the level set framework. Journal of Computational Physics 220(1), 339-354 (2006)

14. Rathi, Y., Vaswani, N., Tannenbaum, A., Yezzi, A.: Tracking deforming objects using particle filtering for geometric active contours. IEEE Trans. Pattern Analysis and Machine Intelligence 29(8), 1470-1475 (2007)

15. Sethian, J.A.: Theory, algorithms, and applications of level set methods for propagating interfaces. Acta Numerica 5(-1), 309-395 (1996) 